\title{
A Ray-Tracing Algorithm for Spinning Compact Object Spacetimes with Arbitrary Quadrupole Moments. I. Quasi-Kerr Black Holes
}

\author{
Dimitrios Psaltis and Tim Johannsen \\ Astronomy and Physics Departments, University of Arizona, 933 N. Cherry Ave., Tucson, AZ \\ 85721, USA
}

\begin{abstract}
We describe a new numerical algorithm for ray tracing in the external spacetimes of spinning compact objects characterized by arbitrary quadrupole moments. Such spacetimes describe non-Kerr vacuum solutions that can be used to test the no-hair theorem in conjunction with observations of accreting black holes. They are also appropriate for neutron stars with spin frequencies in the $\simeq 300-600 \mathrm{~Hz}$ range, which are typical of the bursting sources in low-mass X-ray binaries. We use our algorithm to show that allowing for the quadrupole moment of the spacetime to take arbitrary values leads to observable effects in the profiles of relativistic broadened fluorescent iron lines from geometrically thin accretion disks.
\end{abstract}

Subject headings: black hole physics — radiative transfer — relativity

\section{Introduction}

The observational appearance of accreting black holes and of neutron stars is strongly influenced by gravitational lensing in their exterior spacetimes. In the case of accreting black holes, different aspects of this ray-tracing problem have been addressed by several research groups to date, as applied to the spectra, timing, and images of the accretion flows (e.g., Bardeen 1973; Cunningam 1975; Laor 1991; Rauch \& Blandford 1994; Speith, Riffert, \& Ruder 1995; Dovciak, Karas, \& Yaqoob 2004; Beckwith \& Done 2004; Broderick 2006; Dexter \& Agol 2009; Dolence et al. 2009). In the case of neutron stars, the effect of light bending on the spectra and lightcurves generated by their surface emission has been explored for non-spinning (e.g., Pechenick, Ftaclas, \& Cohen 1983), slowly spinning (Miller \& Lamb 1998; Braje, Romani, \& Rauch 2000; Muno, Özel, \& Chakrabarty 2002; Poutanen \& Beloborodov 2006), and rapidly spinning neutron stars (Cadeau et al. 2007; Morsink et al. 2007).

The external spacetimes of black holes and of slowly spinning neutron stars are described by the Kerr solution. This metric is of Petrov-type D and, therefore, the Carter constant is an integral of motion along the trajectories of photons (Carter 1968). The presence of this integral of motion, in addition to the conservation of energy and angular momentum, allows one to use first-order differential equations to solve for the photon trajectories. This improves the accuracy 
of the calculation and increases its speed (e.g., Rauch \& Blandford 1994; Dexter \& Agol 2009; see, however, Broderick 2006; Dolence et al. 2009 for different approaches).

The Kerr metric is very particular both in the sense that it is completely described by only two parameters (the mass and the spin of the compact object) and that orbits within this metric are characterized by the Carter constant (see, e.g., discussion in Will 2009). Introducing any deviation from the Kerr metric, while satisfying the vacuum Einstein field equations, does not preserve its Petrov-type D character and the Carter constant is no longer conserved along geodesics (see, e.g., Glampedakis \& Babak 2006; Gair, Li, \& Mandel 2008). As a result, ray tracing in a non-Kerr metric cannot be performed entirely using integrals of motions but requires integrating the second-order differential equations for individual geodesics.

There are at least two distinct astrophysical settings for which ray tracing in a metric that deviates from the Kerr solution is important. First, the external spacetime of a neutron star spinning at $\simeq 300-600 \mathrm{~Hz}$, which is typical of X-ray bursters in low-mass X-ray binaries, is not accurately described by the Kerr metric. Effects related to the oblateness of the star (Morsink et al. 2007) as well as to deviations of the quadrupole moment of its spacetime from the Kerr value (Hartle \& Thorne 1968; see also Laarakkers \& Poisson 1999; Berti \& Stergioulas 2004) are not negligible at these spin frequencies. Matching the theoretical models to the level of accuracy reached with current observations of spinning neutron stars can only be achieved by considering at least the deviation of the quadrupole moments of their spacetimes from the Kerr values.

Second, calculating the observational appearance of black holes with arbitrary quadrupole moments can be used in testing the no-hair theorem with astrophysical observations (Johannsen \& Psaltis 2010a, 2010b, 2010c). The absence of additional 'hair' ensures that all moments of a black hole spacetime that are higher than the dipole have a particular dependence on the mass and the spin of the black hole. In particular, the quadrupole moment $q$ of a black hole spacetime has to depend on its spin $a$ according to the relation $q=-a^{2}$, where all quantities have been normalized with appropriate powers of the mass $M$ of the black hole. Allowing for the quadrupole moment of the spacetime to take arbitrary values and using observations to test the validity of the above relation between the quadrupole and the spin of the black hole constitutes a formal quantitative test of the no-hair theorem (Ryan 1995).

Here we present a new ray-tracing algorithm for calculating the observational appearance of spinning compact objects with arbitrary quadrupole moments. We employ the metric of Glampedakis \& Babak (2006), which is characterized by three parameters: the mass and spin of the compact object and its quadrupole moment. In the algorithm, we integrate two first-order differential equations that arise from integrals of motion as well as two second-order differential equations for two components of the geodesic equations in order to compute the trajectories of photons in these spacetimes. We also use a third integral of motion related to the norm of the photon 4-momenta in order to monitor the accuracy of the calculations. In the first paper of this series, we focus on an application related to the line spectra of accreting black holes with spacetimes that violate the 
no-hair theorem.

In $\S 2$, we describe the metric of Glampedakis \& Babak (2006) in some detail and, in $\S 3$, we outline the numerical algorithm. Finally, in $\S 4$, we present some illustrative results for different astrophysical settings while comparing the results of our algorithm with other calculations for Kerr black holes.

\section{A Vacuum Metric with an Arbitrary Quadrupole Moment}

We describe the external spacetime of a spinning compact object with an arbitrary quadrupole moment using the metric of Glampedakis \& Babak (2006). This metric arises by adding to the Kerr solution a contribution that has an arbitrary quadrupole moment and is by itself a solution to the vacuum Einstein field equations (Hartle \& Thorne 1968).

The metric is specified uniquely by three parameters: the mass $M$ of the compact object, the spin $a$, and the deviation $\epsilon$ of its quadrupole moment from the Kerr value. Setting $\epsilon=0$ makes the metric equal to the Kerr solution, which is appropriate for a black hole of arbitrary spin. On the other hand, when $a / M \lesssim 0.4$, all moments of the spacetime that are higher than the quadrupole are negligible and the metric becomes appropriate for neutron stars that are spinning moderately but not close to their mass shedding limit (Hartle \& Thorne 1968).

Following Glampedakis \& Babak (2006), we write the metric in Boyer-Lindquist coordinates as

$$
g_{\mu \nu}=g_{\mu \nu}^{\mathrm{K}}+\epsilon h_{\mu \nu} .
$$

Here $g_{\mu \nu}^{\mathrm{K}}$ is the Kerr metric with the line element

$d s^{2}=-\left(1-\frac{2 M r}{\Sigma}\right) d t^{2}-\left(\frac{4 M a r \sin ^{2} \theta}{\Sigma}\right) d t d \phi+\left(\frac{\Sigma}{\Delta}\right) d r^{2}+\Sigma d \theta^{2}+\left(r^{2}+a^{2}+\frac{2 M a^{2} r \sin ^{2} \theta}{\Sigma}\right) \sin ^{2} \theta d \phi^{2}$.

In this relation,

$$
\Delta \equiv r^{2}-2 M r+a^{2}
$$

and

$$
\Sigma \equiv r^{2}+a^{2} \cos ^{2} \theta
$$

The quadrupole correction is given, in contravariant form, by

$$
\begin{aligned}
h^{t t} & =(1-2 M / r)^{-1}\left[\left(1-3 \cos ^{2} \theta\right) \mathcal{F}_{1}(r)\right], \\
h^{r r} & =(1-2 M / r)\left[\left(1-3 \cos ^{2} \theta\right) \mathcal{F}_{1}(r)\right], \\
h^{\theta \theta} & =-\frac{1}{r^{2}}\left[\left(1-3 \cos ^{2} \theta\right) \mathcal{F}_{2}(r)\right], \\
h^{\phi \phi} & =-\frac{1}{r^{2} \sin ^{2} \theta}\left[\left(1-3 \cos ^{2} \theta\right) \mathcal{F}_{2}(r)\right], \\
h^{t \phi} & =0
\end{aligned}
$$


with the functions $\mathcal{F}_{1,2}(r)$ shown explicitly in Appendix A of Glampedakis \& Babak (2006). If the spacetime of a compact object is described by this solution, its quadrupole moment is

$$
q=-M\left(a^{2}+\epsilon M^{2}\right),
$$

whereas all the higher order moments take their corresponding Kerr values.

Calculating the observational appearance of a black hole that violates the no-hair theorem using the above metric requires a phenomenological scheme to handle its irregularities at $r \simeq 2 M$ (see Glampedakis \& Babak 2006; Gair et al. 2008; Johannsen \& Psaltis 2010a). Precisely because of the no hair theorem, the only axisymmetric vacuum solution to the Einstein field equations that does not contain naked singularities or closed time-like loops is the Kerr metric. Allowing

for deviations of the quadrupole moment while requiring that the spacetime remains a solution to the field equations is necessarily accompanied by the introduction of pathologies to the spacetime. In the case of the metric (11) and for spins $a / M \lesssim 0.4$, these pathologies appear at $r \leq 2.6 M$ (Johannsen \& Psaltis 2010a). We consider these to be unphysical and handle them by removing from the domain of solution all inbound photons that cross $r=2.6 M$. Because, for $a / M \lesssim 0.4$, this radius is smaller than the radius of the photon orbit, we expect that this scheme affects only marginally the images and spectra seen by an observer at infinity.

\section{The Ray Tracing Algorithm}

In this section, we describe the numerical algorithm for the calculation of the trajectories of individual photons from the image plane of an observer at infinity to the location of their emission. Depending on the problem at hand, the latter may be the stellar surface or an accretion disk. Following Cadeau et al. (2007), we use two integrals of motion to write first-order differential equations for the time coordinate and the azimuth of each photon trajectory. We then complete the system using the second-order differential equations for the geodesics along the radial and polar coordinates.

The metric (11) is stationary and axisymmetric. It is, therefore, characterized by the two usual Killing vectors, $\xi=(1,0,0,0)$ and $\eta=(0,0,0,1)$, which correspond to the conservation of energy

$$
E=-g_{t t} \frac{d t}{d \lambda}-g_{t \phi} \frac{d \phi}{d \lambda}
$$

and angular momentum

$$
L=g_{\phi \phi} \frac{d \phi}{d \lambda}+g_{t \phi} \frac{d t}{d \lambda}
$$

along the photon trajectory. Here, $g_{\mu \nu}$ is the $\mu \nu$-element of the metric, and $\lambda$ is an affine parameter. Using these two conserved quantities, we now write two first-order differential equations for the evolution of the $t$ - and $\phi$ - components of the photon position as

$$
\frac{d t}{d \lambda^{\prime}}=\frac{-g_{\phi \phi}-b g_{t \phi}}{g_{\phi \phi} g_{t t}-g_{t \phi}^{2}}
$$


and

$$
\frac{d \phi}{d \lambda^{\prime}}=\frac{b g_{t t}+b g_{t \phi}}{g_{\phi \phi} g_{t t}-g_{t \phi}^{2}}
$$

where we have defined the normalized affine parameter $\lambda^{\prime} \equiv E \lambda$ and the impact parameter for the photon trajectory $b \equiv L / E$.

For the $r$ - and $\theta$ - components of the photon position we use the second-order geodesic equations, which for a general axisymmetric metric take the form

$\frac{d^{2} r}{d \lambda^{\prime 2}}=-\Gamma_{t t}^{r}\left(\frac{d t}{d \lambda^{\prime}}\right)^{2}-\Gamma_{r r}^{r}\left(\frac{d r}{d \lambda^{\prime}}\right)^{2}-\Gamma_{\theta \theta}^{r}\left(\frac{d \theta}{d \lambda^{\prime}}\right)^{2}-\Gamma_{\phi \phi}^{r}\left(\frac{d \phi}{d \lambda^{\prime}}\right)^{2}-2 \Gamma_{\phi t}^{r}\left(\frac{d \phi}{d \lambda^{\prime}}\right)\left(\frac{d t}{d \lambda^{\prime}}\right)-2 \Gamma_{\theta r}^{r}\left(\frac{d \theta}{d \lambda^{\prime}}\right)\left(\frac{d r}{d \lambda^{\prime}}\right)$

and

$\frac{d^{2} \theta}{d \lambda^{\prime 2}}=-\Gamma_{t t}^{\theta}\left(\frac{d t}{d \lambda^{\prime}}\right)^{2}-\Gamma_{r r}^{\theta}\left(\frac{d r}{d \lambda^{\prime}}\right)^{2}-\Gamma_{\theta \theta}^{\theta}\left(\frac{d \theta}{d \lambda^{\prime}}\right)^{2}-\Gamma_{\phi \phi}^{\theta}\left(\frac{d \phi}{d \lambda^{\prime}}\right)^{2}-2 \Gamma_{\phi t}^{\theta}\left(\frac{d \phi}{d \lambda^{\prime}}\right)\left(\frac{d t}{d \lambda^{\prime}}\right)-2 \Gamma_{\theta r}^{\theta}\left(\frac{d \theta}{d \lambda^{\prime}}\right)\left(\frac{d r}{d \lambda^{\prime}}\right)$

Here, $\Gamma_{\beta \gamma}^{\alpha}$ are the various Christoffel symbols for the metric (1).

A final integral of motion arises from the requirement that the norm of the photon 4-momentum has to vanish, i.e.,

$$
g_{t t}\left(\frac{d t}{d \lambda^{\prime}}\right)^{2}+g_{r r}\left(\frac{d r}{d \lambda^{\prime}}\right)^{2}+g_{\theta \theta}\left(\frac{d \theta}{d \lambda^{\prime}}\right)^{2}+g_{\phi \phi}\left(\frac{d \phi}{d \lambda^{\prime}}\right)^{2}+2 g_{t \phi}\left(\frac{d t}{d \lambda^{\prime}}\right)\left(\frac{d \phi}{d \lambda^{\prime}}\right)=0 .
$$

This integral of motion is not useful for replacing either the geodesic equation (11) or (12) because it contains the squares of the derivatives of the $r$ - and $\theta$ - coordinates with respect to the affine parameter. Keeping track of the appropriate sign for the two derivatives, especially near the inflection points of the geodesics, would more than offset the benefit of using a first-order integral of motion as opposed to a second-order geodesic equation. Therefore, following Cadeau et al. (2007), we use this integral of motion only in order to monitor the accuracy of the calculation. To this end, we define the parameter

$$
\xi \equiv\left[g_{r r}\left(\frac{d r}{d \lambda^{\prime}}\right)^{2}+g_{\phi \phi}\left(\frac{d \phi}{d \lambda^{\prime}}\right)^{2}+g_{\theta \theta}\left(\frac{d \theta}{d \lambda^{\prime}}\right)^{2}+2 g_{t \phi}\left(\frac{d t}{d \lambda^{\prime}}\right)\left(\frac{d \phi}{d \lambda^{\prime}}\right)\right] /\left[g_{t t}\left(\frac{d t}{d \lambda^{\prime}}\right)^{2}\right]
$$

and test whether its value remains equal to $\xi=-1$ along each geodesic.

Starting from a fine raster of points on the image plane of an observer at infinity, we follow the geodesics backwards to the surface of the compact object or to different regions in the accretion flow where the photons originate.

Following Johannsen \& Psaltis (2010b), we consider an observer viewing the central object from a large distance $d$ and at an inclination angle $\theta_{o}$ from its rotation axis (see Fig. 1). We set up a virtual image plane that is perpendicular to the line of sight and centered at $\phi=0$ of the spacetime. 


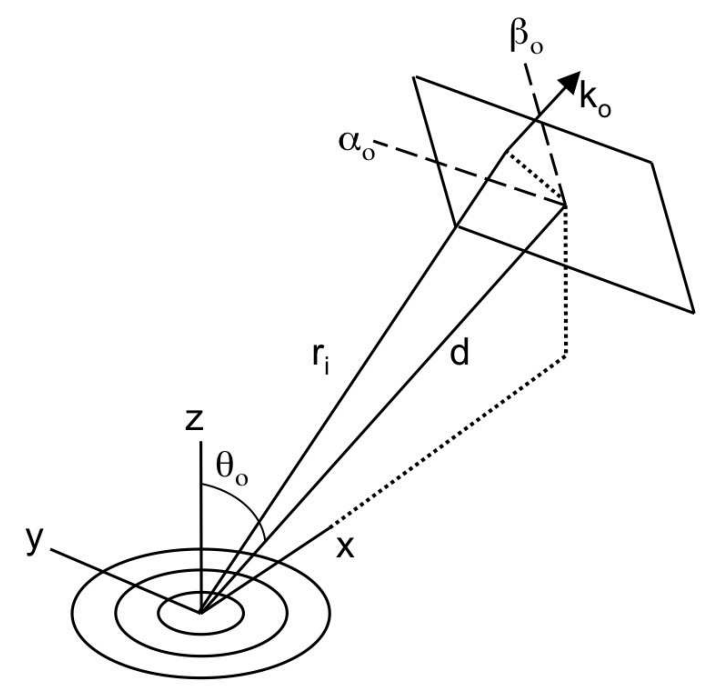

Fig. 1.- The geometry of ray tracing.

We define the set of Cartesian coordinates $\left(\alpha_{0}, \beta_{0}\right)$ on the image plane such that the $\beta_{0}$-axis is along the same fiducial plane and the $\alpha_{0}$-axis is perpendicular to it. We then convert the coordinates $\left(\alpha_{0}, \beta_{0}\right)$ of a photon that reaches the image plane to the coordinates $\left(r_{i}, \theta_{i}, \phi_{i}\right)$ in the spherical-polar system used for the metric (1) with the relations (see Johannsen \& Psaltis 2010b)

$$
\begin{aligned}
r_{i} & =\left(d^{2}+\alpha_{0}^{2}+\beta_{0}^{2}\right)^{1 / 2} \\
\cos \theta_{i} & =\frac{1}{r_{i}}\left(d \cos \theta_{o}+\beta_{0} \sin \theta_{o}\right) \\
\tan \phi_{i} & =\alpha_{0}\left(d \sin \theta_{o}-\beta_{0} \cos \theta_{o}\right)^{-1} .
\end{aligned}
$$

The photons that contribute to the image of the compact object are those with 3-momenta that are perpendicular to the image plane. This orthogonality condition uniquely specifies the momentum vector of a photon with the above coordinates, according to the relations (Johannsen \& Psaltis 2010b)

$$
\begin{aligned}
k^{r} & \equiv \frac{d r}{d \lambda^{\prime}}=\frac{d}{r_{i}} \\
k^{\theta} & \equiv \frac{d \theta}{d \lambda^{\prime}}=\left[-\cos \theta_{o}+\frac{d}{r_{i}^{2}}\left(d \cos \theta_{o}+\beta_{0} \sin \theta_{o}\right)\right]\left[r_{i}^{2}-\left(d \cos \theta_{o}+\beta_{0} \sin \theta_{o}\right)^{2}\right]^{-1 / 2} \\
k^{\phi} & \equiv \frac{d \phi}{d \lambda^{\prime}}=\frac{-\alpha_{0} \sin \theta_{o}}{\left(d \sin \theta_{o}-\beta_{0} \cos \theta_{o}\right)^{2}+\alpha_{0}^{2}} .
\end{aligned}
$$

Using these relations, we then calculate the $t$-component of the photon 4-momentum from equation (13). At this point, the normalization of the photon 4-momentum is arbitrary. Note that, 


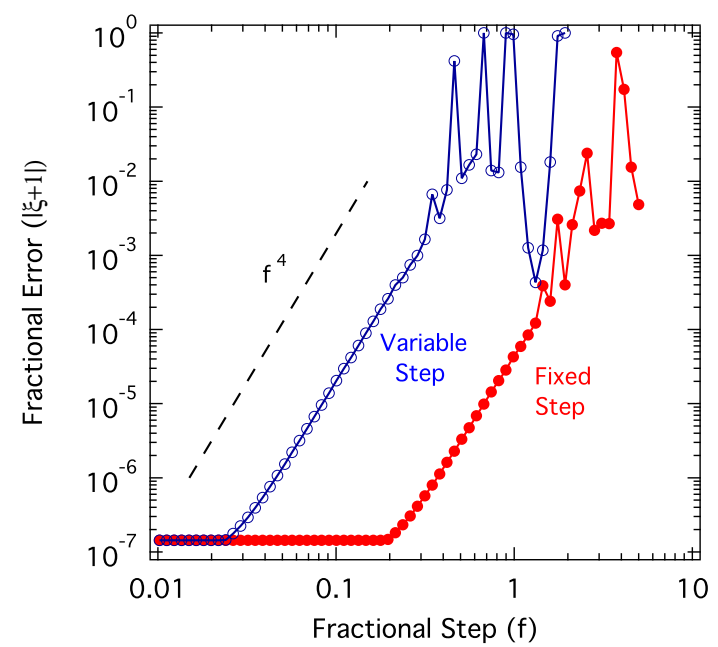

Fig. 2. - The fractional error introduced by the integration algorithm during the tracing of a single ray from the image plane to the equatorial plane, as a function of the fractional stepsize of the integration. The image plane is set at a distance of $1028 M$ and at an inclination of $40^{\circ}$. The black hole spin is equal to $a / M=0.5$ and its spacetime is described by the Kerr metric. The Cartesian coordinates of the position of the ray on the image plane are $\left(\alpha_{0}, \beta_{0}\right)=(5,0)$. The results of two integrations are shown, one with a fixed step in the affine parameter and one with a variable step. In both cases, the performance of the algorithm is consistent with the fourth-order of the integration method.

for a distant observer, $d / r_{i} \rightarrow 1$, which implies that special care needs to be taken in evaluating expressions (19) and (20) to avoid round-off errors.

In the numerical algorithm, we integrate equations (99)-(12) using a fourth-order Runge-Kutta integrator. Figure 2 shows the convergence of our algorithm, for two cases. In the first case, we employ a fixed step $f$ in the affine parameter. In the second case, we employ an adaptive stepsize that we set equal to a fixed fraction $f$ of the inverse rate of the fastest changing variable at each point, i.e.,

$$
\delta \lambda^{\prime}=f \min \left[t\left(\frac{d t}{d \lambda^{\prime}}\right)^{-1}, r\left(\frac{d r}{d \lambda^{\prime}}\right)^{-1}, \theta\left(\frac{d \theta}{d \lambda^{\prime}}\right)^{-1}, \phi\left(\frac{d \phi}{d \lambda^{\prime}}\right)^{-1}\right] .
$$

In both cases, the algorithm shows the expected fourth-order convergence of our integration method. For very small values of the step size, the fractional error stabilizes and reflects the numerical accuracy to which the parameter $\xi$ was calculated on the image plane. For the calculations shown hereafter, we will use an adaptive step with $f=1 / 32 \simeq 0.03$.

Our numerical algorithm is capable of integrating $\simeq 10^{4}$ geodesics in a time comparable to a second, on a personal computer with a $2.5 \mathrm{GHz}$ Intel core. This is comparable to the speed of other similar algorithms that employ different methods (e.g., Dexter \& Agol 2009; Doelence et al. 2009). Moreover, the algorithm is trivially parallelizable and, because of its very low storage requirements, 
is optimal for implementation on a GPU.

\section{Relativistically Broadened Fluorescent Lines Around Quasi-Kerr Black Holes}

As a first application of our numerical algorithm, we calculate the profiles of relativistic broadened fluorescent iron lines from geometrically thin accretion disks around quasi-Kerr black holes. These are prime targets for current and future X-ray telescopes and are expected to lead to the measurements of the spins of a large number of black holes in binary systems and in active galactic nuclei (for reviews see Reynolds \& Nowak 2003; Miller 2007).

For this application, we trace rays from the image plane to the equatorial plane, where we will assume that a geometrically thin disks exists, from some outer radius $r_{\text {out }}$ down to the radius of the innermost stable circular orbit (ISCO).

The character of the ISCO in the metric (1) depends on the sign and magnitude of the quadrupole deviation parameter $\epsilon$. When $\epsilon>0$, orbits close to the central object become unstable to radial perturbations. Following Johannsen \& Psaltis (2010c), we calculate the location of the ISCO in this case by finding the zero in the radial profile of the square of the radial epicyclic frequency for a particle in a circular equatorial orbit. For values of the quadrupole deviation parameter $\epsilon$ that are sufficiently negative, all circular equatorial orbits become stable to radial perturbations. However even in this case, orbits very close to the central object become unstable to vertical perturbations (see also Gair et al. 2008). Presently, we consider only positive deviations of the quadrupole moment of the metric from the Kerr value, i.e., $\epsilon>0$. When $\epsilon=0$, we use the complete expression for the location of the ISCO from Bardeen et al. (1973).

We assume that the disk is composed of a set of equatorial concentric rings, in which the plasma is moving at the local Keplerian velocity with (Glampedakis \& Babak 2006)

$$
\begin{aligned}
u^{\phi} & =\frac{1}{\Delta}\left[\frac{2 M}{r}(a E-L)+L\right]-\epsilon \frac{h_{3}}{r^{2}} L \\
u^{t} & =\frac{1}{\Delta}\left[E\left(r^{2}+a^{2}\right)+\frac{2 M a}{r}(a E-L)\right]-\epsilon f_{3} E\left(1-\frac{2 M}{r}\right)^{-1} .
\end{aligned}
$$

Here, $E$ and $L$ are the energy and angular momentum of the circular orbit at radius $r$ and are given in Johannsen \& Psaltis (2010a).

We also assume that radiation emerges from the accretion disk surface isotropically with an "emissivity" that scales as the power-law function $r^{-\alpha}$ of the coordinate radius. We calculate the overall change in the energy of the photon from the location of emission at the accretion disk to the image plane using

$$
\frac{E_{\mathrm{im}}}{E_{\mathrm{d}}}=\frac{g_{\mu \nu, \mathrm{im}} k_{\mathrm{im}}^{\mu} u_{\mathrm{im}}^{\nu}}{g_{\mu \nu, \mathrm{d}} k_{\mathrm{d}}^{\mu} u_{\mathrm{d}}^{\nu}}
$$

and setting the 3 -velocity of the observer at the image plane to zero. In this last expression, the 


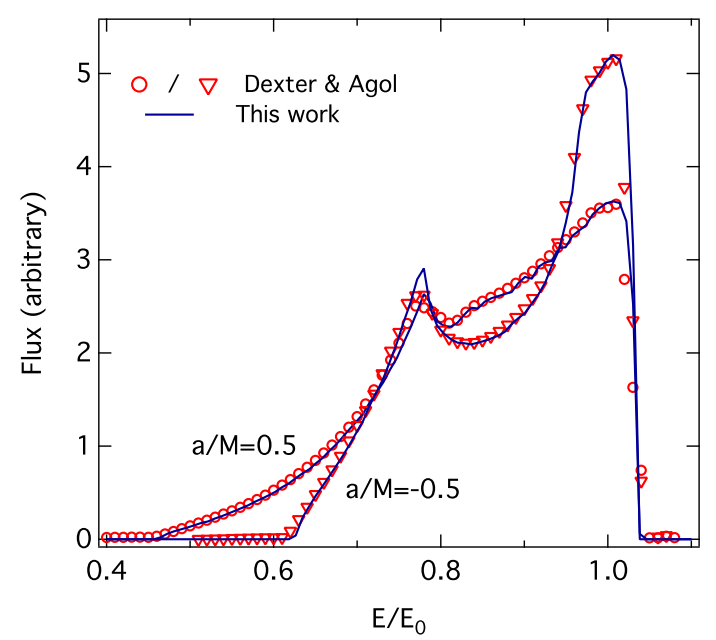

Fig. 3.- Profiles of relativistically broadened fluorescent lines from a geometrically thin accretion disk around a Kerr black hole, for two values of the black-hole spin. The outer radius of the disk is set to $15 M$, the power-law index of the line emissivity to $\alpha=2$, and the inclination to the observer to $\theta_{\mathrm{o}}=30^{\circ}$. The symbols are the results reported by Dexter \& Agol (2009), while the solid lines are the profiles calculated with the algorithm described here.

subscripts "im" and "d" refer to the image plane and the accretion disk, respectively. We finally employ the Lorentz invariant quantity $I / E^{3}$, where $I$ is the monochromatic specific intensity of the radiation field and $E$ is the photon energy, in order to calculate the specific intensity at each point on the image plane.

The result at the completion of this calculation is the overall redshift or blueshift experienced by a photon that reaches each point on the image plane, which we denote by $g\left(\alpha_{0}, \beta_{0}\right) \equiv E_{\mathrm{im}} / E_{\mathrm{d}}$, and the corresponding specific intensity $I\left(\alpha_{0}, \beta_{0}\right)$. The monochromatic flux at the image plane is then

$$
F_{E} \sim \frac{1}{d^{2}} \int d \alpha_{0} \int d \beta_{0} I\left(\alpha_{0}, \beta_{0}\right) \delta\left[E-E_{0} g\left(\alpha_{0}, \beta_{0}\right)\right] .
$$

The presence of the $\delta$-function allows us in principle to convert this 2-dimensional integral into a one-dimensional line integral along contours of constant values of the quantity $g\left(\alpha_{0}, \beta_{0}\right)$ on the image plane. In practice, calculating the location of these contours is time consuming. Instead, we evaluate expression (25) using a Monte Carlo integration of points on the image plane and a set of fine bins in photon energy.

Figures 3 and 4 show the dependence of the line profiles on the spin of the black hole and on the emissivity of fluorescence on the accretion disk. They also compare our results with other calculations from the literature, demonstrating the agreement between the different numerical algorithms. Figure 5 shows the dependence of the fluorescent line profiles on the parameter $\epsilon$, which measures the deviation of the quadrupole moment of the black-hole spacetime from its Kerr value. As we 


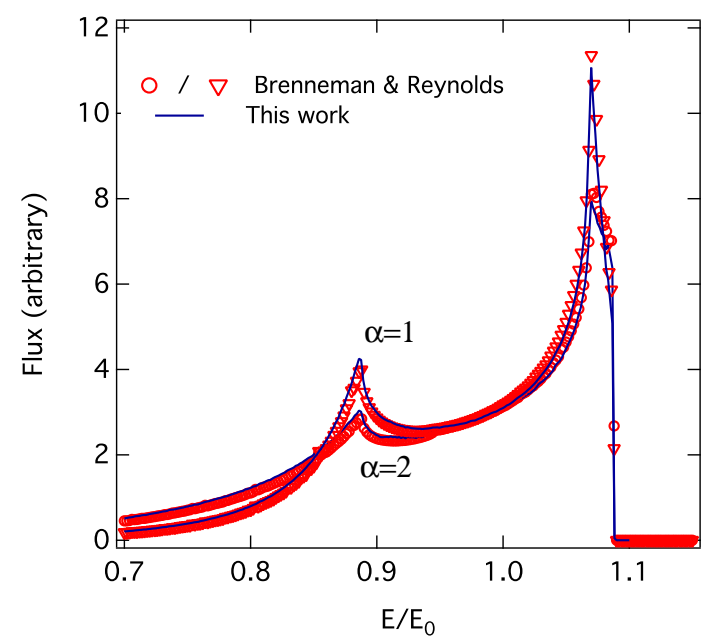

Fig. 4.- Profiles of relativistically broadened fluorescent lines from a geometrically thin accretion disk around a Kerr black hole, for two values of the line emissivity. The black hole spin is equal to $a / M=0.5$, the outer radius of the disk is set to $50 M$, and the inclination to the observer to $\theta_{\mathrm{o}}=40^{\circ}$. The symbols are the results reported by Brenneman \& Reynolds (2006), while the solid lines are the profiles calculated with the algorithm described here.

increase the degree of quadrupole deviation, the red wing of the line becomes less pronounced and the relative strengths of the blue and red wings is altered. Both effects are predominantly caused by the increase in the radius of the ISCO with increasing value of the parameter $\epsilon$.

\section{Conclusions}

We described a new ray tracing algorithm for the calculation of observables from compact objects with spacetimes characterized by arbitrary quadrupole moments. Such spacetimes are relevant to black holes that violate the no-hair theorem and to moderately spinning neutron stars. We put special care in streamlining and accelerating our algorithm in order to achieve the efficiency neccessary for large parameter studies and comparisons to data. We also demonstrated the expected convergence of our algorithm and verified our results against those of previous calculations for Kerr metrics.

As a first application, we calculated the profiles of fluorescent iron lines from black holes that violate the no-hair theorem. As expected, varying the quadrupole moment of the spacetime, led to changes in the detailed profiles of the lines. We will study the observability of these effects as well as ways of breaking the degeneracy between changing the spin and the quadrupole of the black hole in a forthcoming paper. 


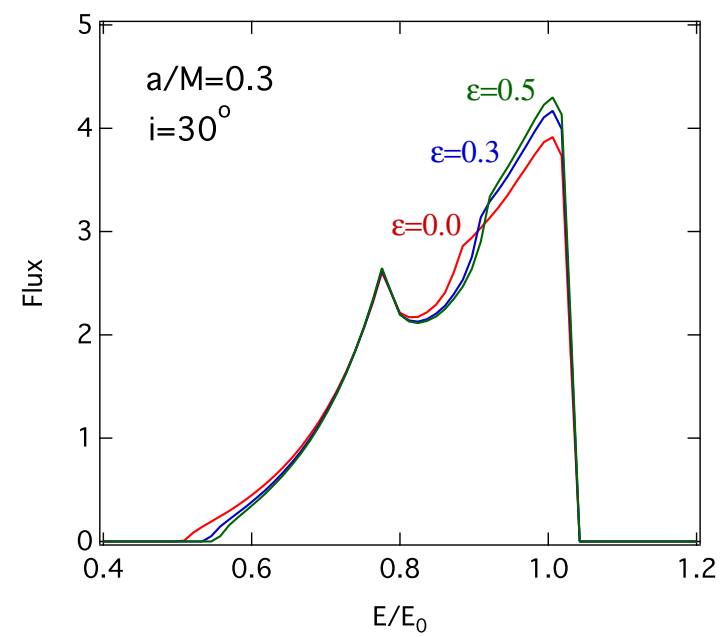

Fig. 5.- Profiles of relativistically broadened fluorescent lines from a geometrically thin accretion disk around a quasi-Kerr black hole, for a spin of $a / M=0.3$ and three values of the quadrupole deviation parameter $\epsilon$. The remaining parameters are the same as in Figure 3 ,

\section{REFERENCES}

Bardeen, J. M. 1973, Black Holes (Les Astres Occultes), 215

Beckwith, K. \& Done, C. 2004, MNRAS 352, 353

Beckwith, K. \& Done, C. 2005, MNRAS 359, 1217

Berti, E., \& Stergioulas, N. 2004, MNRAS, 350, 1416

Braje, T. M., Romani, R. W., \& Rauch, K. P. 2000, ApJ, 531, 447

Brenneman, L. W., \& Reynolds, C. S. 2006, ApJ, 652, 1028

Broderick, A. E. 2006, MNRAS, 366, L10

Broderick, A. E., et al. 2009, ApJ, 697, 45

Cadeau, C., Morsink, S. M., Leahy, D., \& Campbell, S. S. 2007, ApJ, 654, 458

Carter, B. 1968, Phys. Rev., 174, 1559

Collins, N. A., Hughes, S. A. 2004, PRD 69, 124022

Cunningham, C. T. 1975, ApJ, 202, 788

Dexter, J. \& Agol, E. 2009, ApJ 696, 1616

Dexter, J., Agol, E., \& Fragile, P. C. 2009, ApJ, 703, L142 
Doeleman, S. S., et al. 2008, Nature, 455, 78

Dolence, J. C., Gammie, C. F., Mościbrodzka, M., \& Leung, P. K. 2009, ApJS, 184, 387

Dovčiak, M., Karas, V., \& Yaqoob, T. 2004, ApJS, 153, 205

Gair, J. R., Li, C., \& Mandel, I. 2008, Phys. Rev. D, 77, 024035

Glampedakis, K., Babak, S. 2006, CQG 23, 4167

Hartle, J. B., \& Thorne, K. S. 1968, ApJ, 153, 807

Johannsen, T., \& Psaltis, D. 2010a, ApJ, 716, 187

2010b, ApJ, 718, 446

2010c, ApJ, in press

Laarakkers, W. G., \& Poisson, E. 1999, ApJ, 512, 282

Laor, A. 1991, ApJ, 376, 90

Manko, V. S. \& Novikov, I. D. 1992, Class. Quantum Grav. 9, 2477

Miller, J. 2007, ARA\&A, 45, 441

Miller, M. C., \& Lamb, F. K. 1998, ApJ, 499, L37

Morsink, S. M., Leahy, D. A., Cadeau, C., \& Braga, J. 2007, ApJ, 663, 1244

Mościbrodzka, M., Gammie, C. F., Dolence, J. C., Shiokawa, H., \& Leung, P. K. 2009, ApJ, 706, 497

Muno, M. P., Özel, F., \& Chakrabarty, D. 2002, ApJ, 581, 550

Noble, S. C., Krolik, J. H., \& Hawley, J. F. 2010, ApJ, 711, 959

Özel, F., \& Psaltis, D. 2003, ApJ, 582, L31

Pechenick, K. R., Ftaclas, C., \& Cohen, J. M. 1983, ApJ, 274, 846

Poutanen, J., \& Beloborodov, A. M. 2006, MNRAS, 373, 836

Rauch, K. P., \& Blandford, R. D. 1994, ApJ, 421, 46

Reynolds, C., \& Nowak, M. 2003, Phys. Rep., 377, 389

Ryan, F. D. 1995, Phys. Rev. D, 52, 5707

Shibata, M., \& Sasaki, M. 1998, Phys. Rev. D, 58, 104011 
Speith, R., Riffert, H., \& Ruder, H. 1995, Computer Physics Communications, 88, 109

Will, C. M. 2009, Phys. Rev. Lett., 102, 061101 